\title{
Surfactant effects on the dynamics of capillary rise and finger formation in square capillaries
}

Rozeline Wijnhorst, Thijs Christiaan de Goede, Daniel Bonn, and Noushine

$$
\text { Shahidzadeh* }
$$

van der Waals-Zeeman Institute, Institute of Physics, University of Amsterdam, Science Park 904, 1098XH Amsterdam, the Netherlands

\author{
E-mail: n.shahidzadeh@uva.nl
}




\section{Supplementary Image}

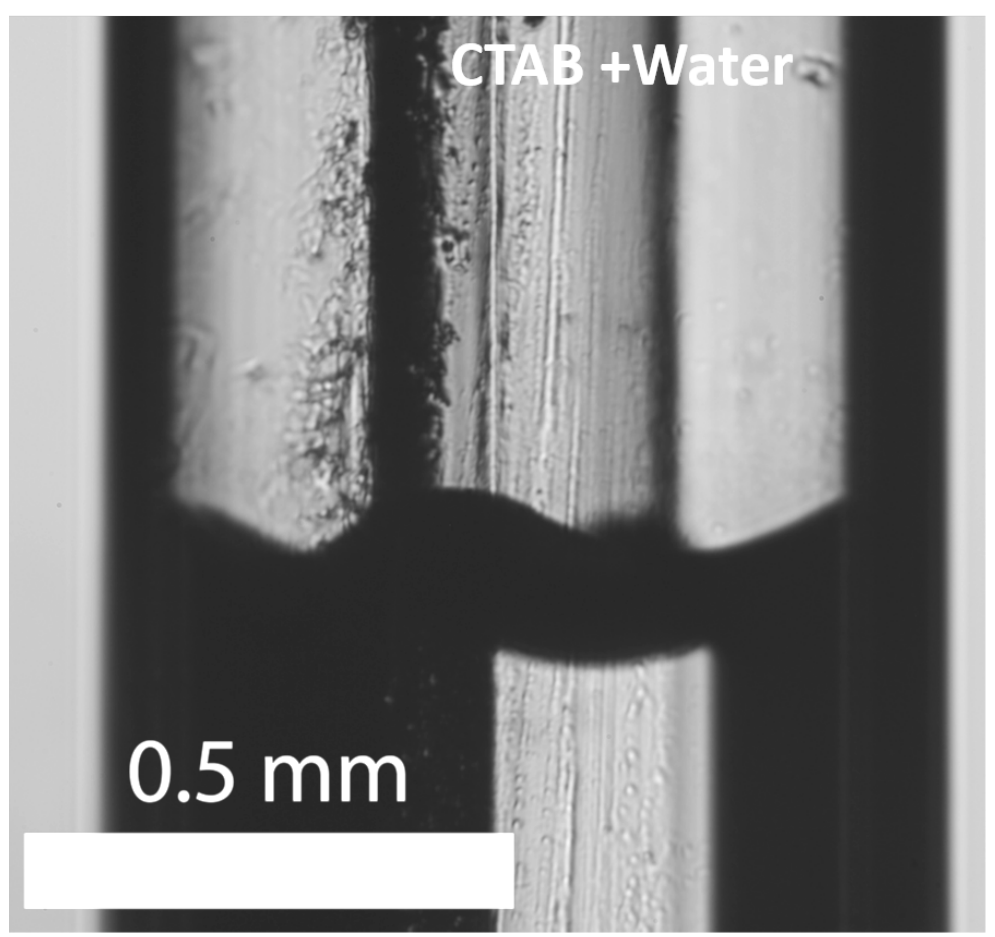

Figure S1: meniscus of the $1 \mathrm{CMC}$ CTAB solution in a borosilicate square capillary (inner diameter $=0.5 \mathrm{~mm}$ ) at equilibrium.

\section{Supplementary Videos}

\section{Supplementary Video 1}

Video of a fluorescent image sequence of a square capillary (inner side $=0.5 \mathrm{~mm}$ ) in contact with water with fluorescent dye (dinartum-2,2'-([1,1'-bifenyl]-4,4'diyldivinyleen)bis, concentration: $0.5 \mathrm{~g} / \mathrm{l}$, purchased from Tuhamij B.V.). Recorded with a Nikon D5300 camera at $50 \mathrm{fps}$. 


\section{Supplementary Video 2}

Video of a fluorescent image sequence of a square capillary (inner side $=0.5 \mathrm{~mm}$ ) in contact with dodecane with fluorescent dye (perylene, mass percentage: 1, from Sigma Aldrich). Recorded with a Nikon D5300 camera at 50 fps.

\section{Supplementary Video 3}

Video of a fluorescent image sequence of a square capillary (inner side $=0.5 \mathrm{~mm}$ ) in contact with a 1 CMC CTAB solution with fluorescent dye (dinartum-2,2'-([1,1'-bifenyl]4,4'diyldivinyleen)bis, concentration: $0.5 \mathrm{~g} / \mathrm{l}$, purchased from Tuhamij B.V.). Recorded with a Nikon D5300 camera at 50 fps.

\section{Supplementary Video 4}

Video of a fluorescent image sequence of a square capillary (inner side $=0.5 \mathrm{~mm}$ ) in contact with a 1 CMC SDS solution with fluorescent dye (dinartum-2,2'-([1,1'-bifenyl]4,4'diyldivinyleen)bis, concentration: $0.5 \mathrm{~g} / \mathrm{l}$, purchased from Tuhamij B.V.). Recorded with a Nikon D850 camera at 30 fps.

\section{Adsorption of CTAB on Hellmanex-III cleaned glass}

The interaction of the CTAB solution with the glass surface can be investigated experimentally using ultraviolet-visible light (UV-Vis) spectrometry and a cationic dye (Methylene Blue MB / C16H18ClN3S, at 8mg/L). Methylene Blue has a maximum absorbance band at $660-670 \mathrm{~nm}^{-1}$ and is widely used for the determination of both surface areas and exchange capacities of clay minerals. Indeed, in contact with a negatively charged surface, the cationic dye will interact and induce the discoloration of the dye solution, which can be measured with Uv-vis spectrometry. Here, we used monodisperse borosilicate glass beads (diameter $200 \sim 250 \mu \mathrm{m}$ ) that were cleaned following the same protocol used for the microcapillaries 


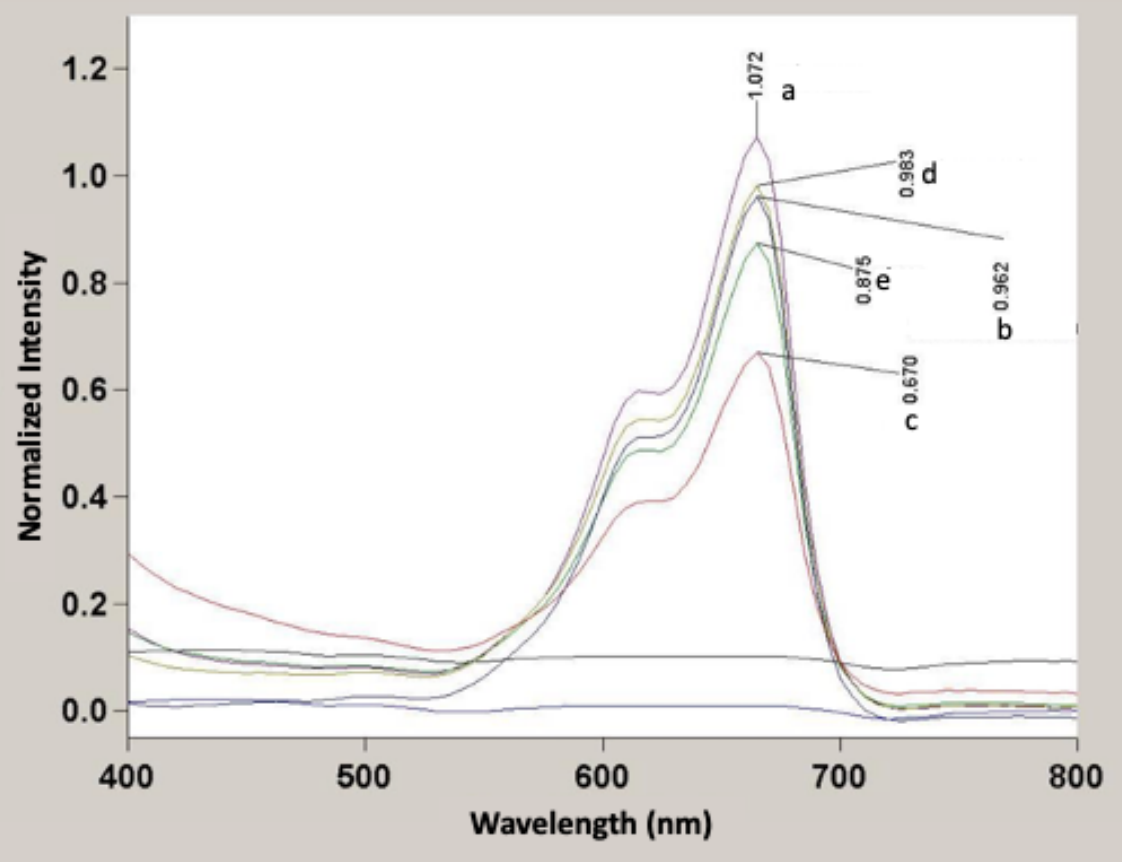

Figure S2: Uv-vis spectrometry on (a) Methylene Blue reference solution (8mg/l) ; (b) and $(\mathrm{d})$ : reference solutions after the addition of cleaned glass beads which were previously immersed in CTAB solution ( $\triangle \mathrm{Abs} \sim 2 \%$ (c): reference solution after the addition of cleaned glass beads $(\mathrm{Abs}=33 \%)$..

as described in the Methods section of the paper.

The clean glass beads $(250 \mathrm{mg})$ were first immersed in water or in the CTAB solution (1 CMC) for 30 minutes, and were then removed from the solution and immersed into the Methylene Blue solution. The absorbance peak of these glass beads mixed with the Methylene Blue solution is then measured using Uv-vis spectrometry (Figure S2), and compared to a reference Methylene Blue solution. Our results show that the Methylene Blue molecules adsorb to the negatively charged cleaned glass beads (c). This turns the dye solution from a bright blue color to being transparent with a reduction of the absorbance by $\Delta \mathrm{AB} \sim 33 \%$ (c). However, if we immerse the glass beads in a 1 CMC CTAB solution (cationic surfactant) and adding them afterwards to the Methylene Blue solution. The reduction in intensity of the absorbance spectrum is significantly lower : $\triangle \mathrm{AB} \sim 2 \%(\mathrm{~b}, \mathrm{~d})$. The beads are much less 
sensitive to the adsorption of the cationic Methylene blue dye because the negative charge of the glass beads surface has already been partly neutralised by the adsorption of the cationic surfactant CTAB during the 30 minutes immersion in the CTAB solution. As SDS is negatively charged it is not possible to measure its adsorption in this way. Using the cationic dye Methylene Blue, the same graph as for water would be obtained.

\section{Surface Tension impure and pure SDS}

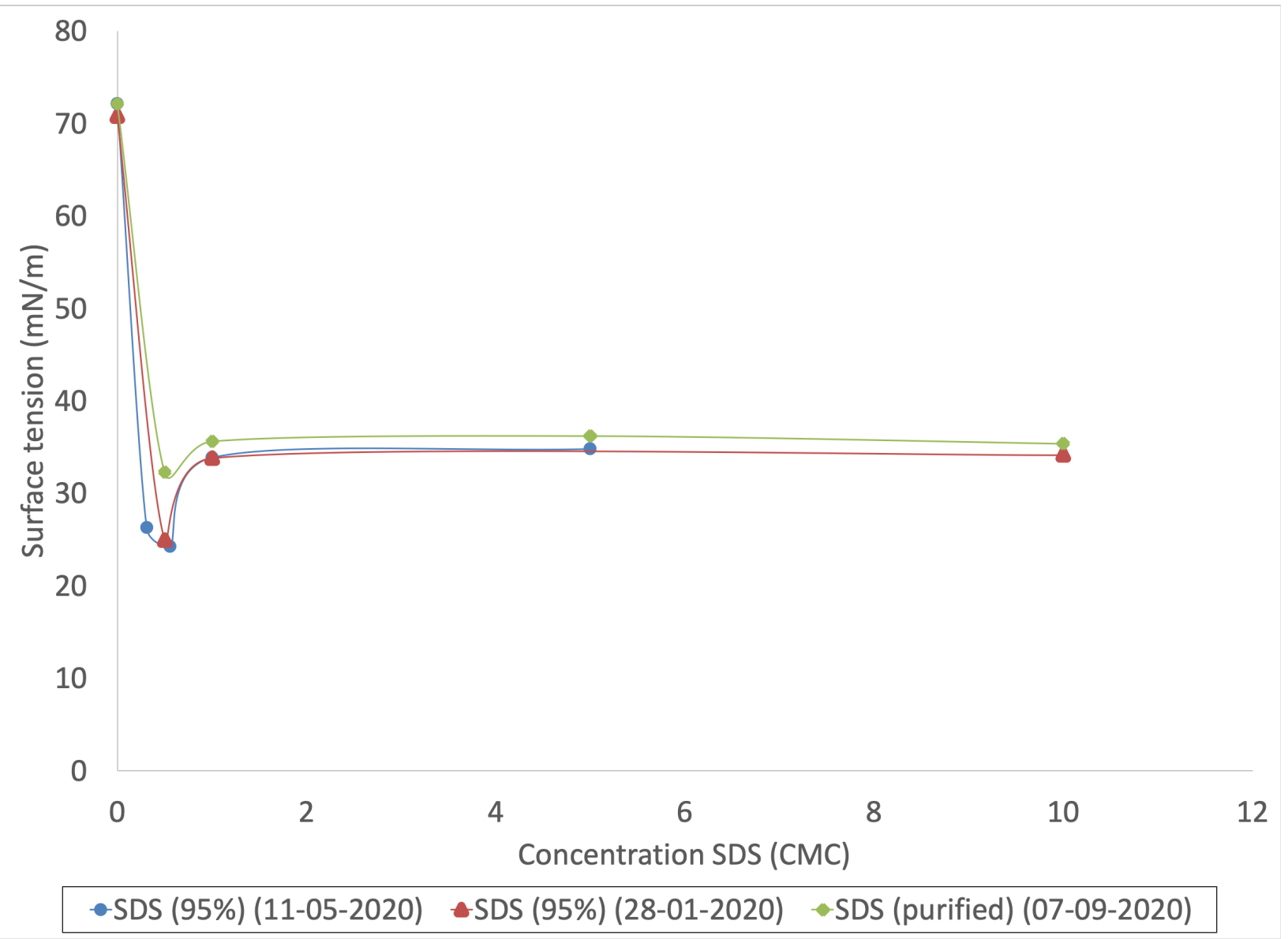

Figure S3: Surface tension versus SDS concentration expressed in CMC. The blue and red datapoints are SDS solutions made of $95 \%$ pure SDS, the green datapoints represent SDS solutions made with purified SDS.

To investigate the influence of impurities on the capillary rise dynamics, we made a comparison between the dynamic surface tension of purified and impurified SDS solutions 


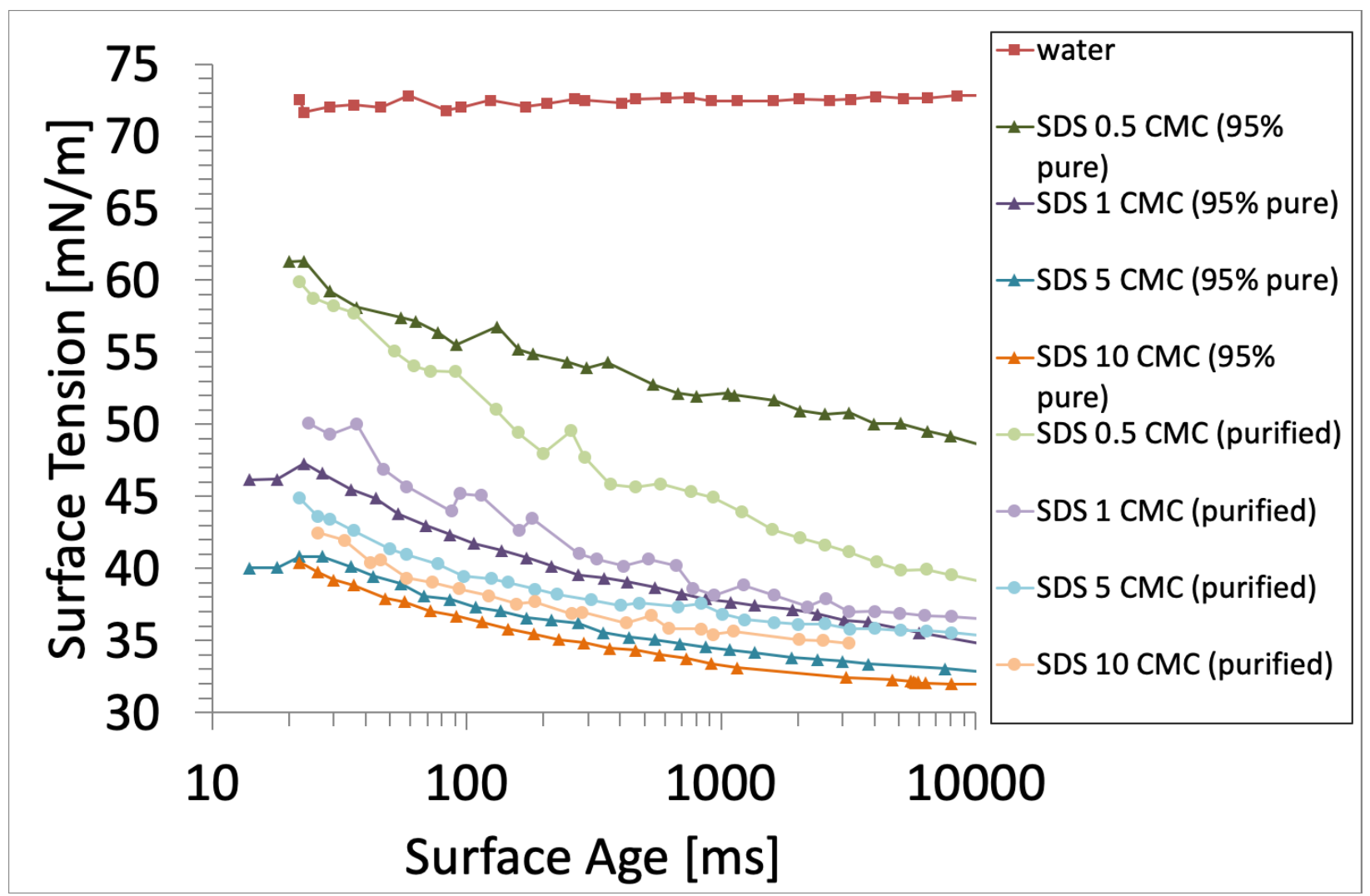

Figure S4: Surface tension $(\mathrm{mN} / \mathrm{m})$ versus Surface age on log-lin scale $(\mathrm{ms})$. The red symbols are the datapoints for water, the green symbols represent 0.5 CMC SDS solutions, the purple symbols represent 1 CMC SDS solutions, the blue symbols represent 5 CMC SDS solutions and the orange symbols represent $10 \mathrm{CMC}$ SDS solutions. The triangles are used for the 95\% pure SDS and the filled circles are used for the purified SDS.

and an isotherm of impure and pure SDS. The SDS was purified using a recrystallization method, where the $95 \%$ pure SDS is used to make a supersaturated solution in ethanol. The recrystallized SDS crystals are used to make aqueous solutions at 0.5 CMC, 1 CMC, 5 CMC and 10 CMC. The same concentrations as been used in the experiments.

The recrystallization reduced the surface tension dip for the $0.5 \mathrm{CMC}$ solution significantly as can be seen in Figure S3. This confirms that the surface tension dip under CMC for impure SDS solutions is due to the impurities.

The comparison between the impure and pure SDS solutions shows that the dynamic surface tension is slightly influenced by the impurities at longer timescales (see Figure S4). The dynamic surface tension of both the 0.5 CMC SDS solutions is in between the surface tension of 
water and the surface tension of SDS solutions above the CMC and decreases with increasing surface age. The Surface age corresponding to the measured equilibrium height is 5000 ms (see Figure S3). At this surface age the difference in dynamic surface tension between impure and pure SDS is roughly $10 \mathrm{mN} / \mathrm{m}(50 \mathrm{mN} / \mathrm{m}$ for the impure SDS and $40 \mathrm{mN} / \mathrm{m}$ for the pure SDS). As the surface tension of the pure SDS decreases faster for increasing surface age, the predicted equilibrium height for a pure 0.5 CMC SDS solution is lower. Although the impurities do seem to influence the dynamic surface tension under CMC, this difference is not seen on this scale above CMC.

Furthermore, the difference in dynamic surface tension could influence the dynamics of the finger growth for impure SDS under the CMC. This difference with pure SDS is not big enough to explain the fast finger growth seen in our experiments. 


\section{Pictures fingertip SDS}

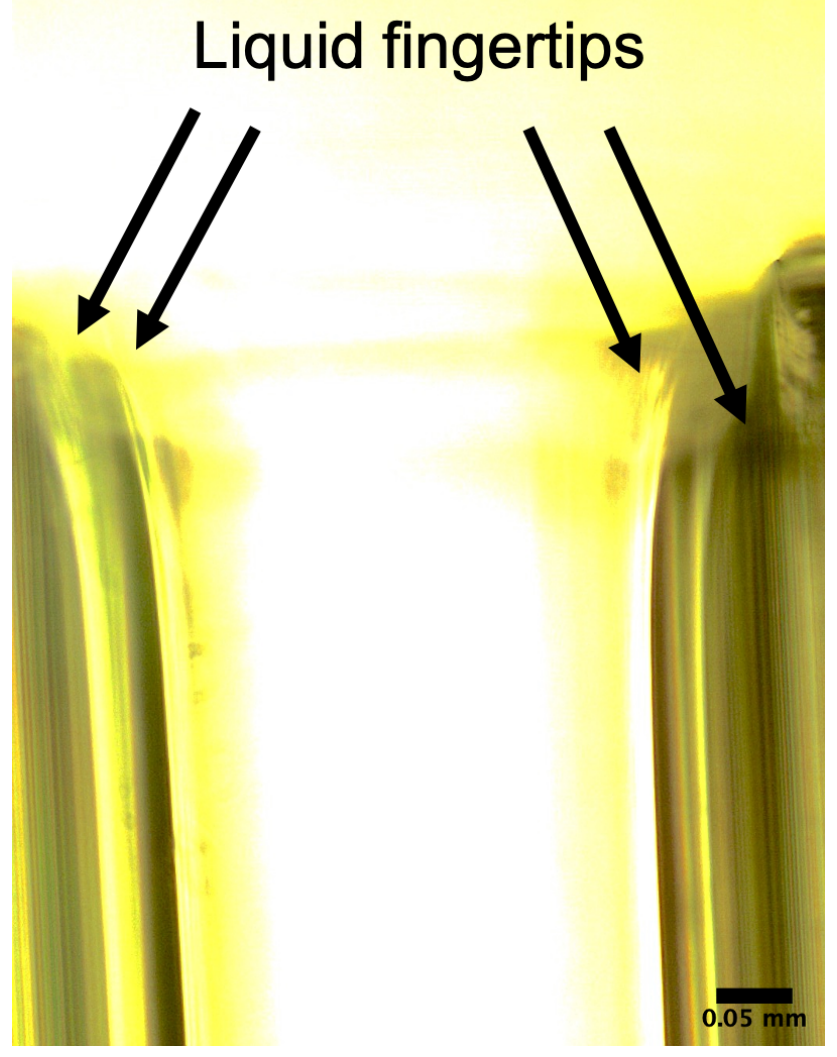

Figure S5: Four fingertips of a 1 CMC SDS solution at the end of a square capillary (inner diameter $=0.5 \mathrm{~mm}$ ) that was horizontally placed so there is no influence of gravity on the shape of the fingertip. 


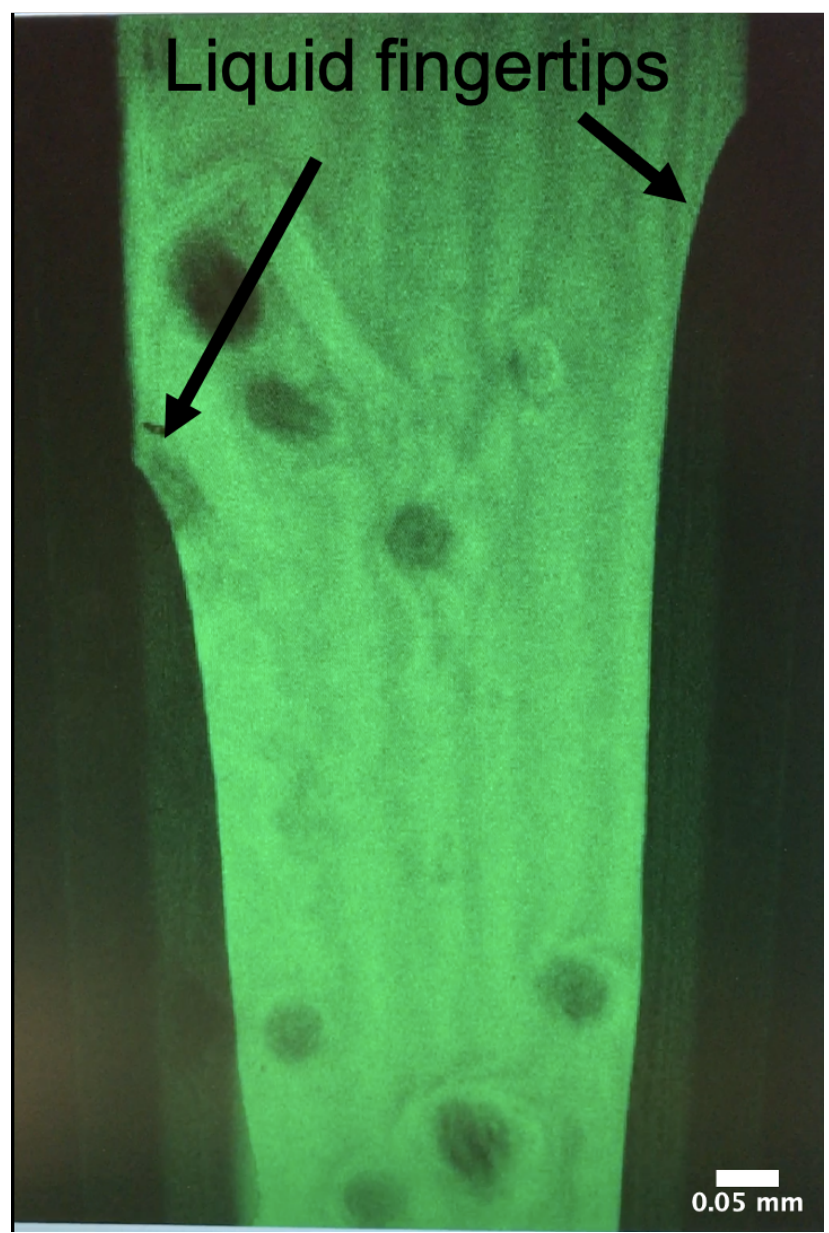

Figure S6: Two fingertips of a 1 CMC SDS solution in a square capillary (inner diameter $=0.5 \mathrm{~mm}$ ). The picture is retrieved using a confocal microscope. The capillary was placed horizontally so there is no influence of gravity on the shape of the fingertip. 

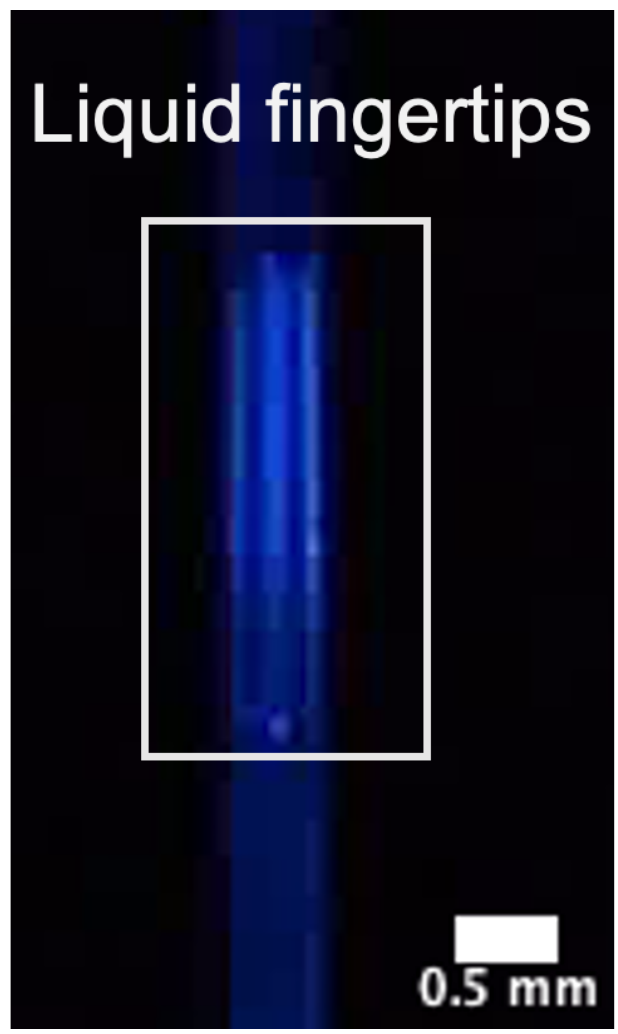

Figure S7: Fingertips of a 1 CMC SDS solution advancing in a vertically placed square capillary (inner diameter $=0.5 \mathrm{~mm}$ ). Fluorescent dye is used to visualize the corner flow, the fingertips have a higher intensity than the lower part of the fingers. 